\title{
Uncovered stent implantation in complicated acute aortic dissection type $B$
}

\author{
Alexander Massmann, MD, ${ }^{\mathrm{a}}$ Takashi Kunihara, PhD, MD,${ }^{\mathrm{c}}$ Peter Fries, MD, \\ Günther Schneider, PhD, MD, ${ }^{\mathrm{a}}$ Arno Buecker, MD, ${ }^{\mathrm{a}}$ and Hans-Joachim Schäfers, $\mathrm{MD}^{\mathrm{b}}$
}

Objective: To retrospectively evaluate the technical feasibility and midterm results of uncovered thoracoabdominal stent placement in complicated acute aortic dissection Stanford type B (cAADB).

Patients and Methods: Fourteen consecutive patients ( 3 females; range, 44-71 years) with cAADB who had symptomatic gastrointestinal malperfusion and claudication underwent immediate uncovered stent implantation (diameter, 7-28 mm; length, 40-100 mm) into the true lumen of the thoracoabdominal aorta $(\mathrm{n}=23)$ and visceral arteries $(\mathrm{n}=5)$.

Results: Stenting resulted in elimination of gastrointestinal ischemia and symptoms in 13 of 14 patients; persisting symptoms led to secondary surgical revascularization in only 1 patient. More than 1 stent $(\leq 4)$ was placed in 7 patients ( 2 celiac, 1 mesenteric, 2 renal, 8 aorto-iliac). Follow-up computed tomographic angiography (CTA) revealed collapse of 4 aortic stents (diameter, $9-25 \mathrm{~mm}$; length, $100 \mathrm{~mm}$ ) at 1 week in the absence of symptoms. Balloon reexpansion was possible in all 4 stents, but recollapse occurred within 1 month. Despite stent collapse, the patients remained asymptomatic; ultrasonography and CTA documented sufficient perfusion of the visceral arteries in all patients. Follow-up ranged from 6 months to 5 years (average, 2.5 years). Except for the patient who underwent iliacomesenteric bypass for unspecific abdominal pain, no other patient required additional interventional or surgical therapy.

Conclusions: Acute aortic dissection with suspicion of visceral ischemia should prompt for immediate intervention. Thoracoabdominal uncovered stent implantation is a technically feasible and effective minimally invasive approach that provided successful relief of acute visceral ischemia and claudication in cAADB. Stent size should be less than the normal aortic diameter to avoid possible stent collapse. (J Thorac Cardiovasc Surg 2014;148:3003-11)

See related commentary on pages 3012-3.

Acute aortic dissection type $\mathrm{B}$ is commonly treated conservatively in the absence of complications. Complicated acute aortic dissection Stanford type B (cAADB) is associated with challenging visceral or peripheral malperfusion, which is clinically apparent by abdominal pain caused by (small-) bowel ischemia or renal infarction. Untreated or delayed treatment beyond the duration of visceral ischemia tolerance results in death. The main goal is to resolve ischemia as soon as possible.

Traditionally, this scenario has been treated by replacement of the descending aorta, fenestration procedures, and bare-metal stent implantation. Interventional fenestration

\footnotetext{
From the Clinic of Diagnostic and Interventional Radiology, ${ }^{\mathrm{a}}$ and Clinic of Thoracic and Cardiovascular Surgery, ${ }^{b}$ Saarland University Medical Center, Homburg, Germany; and The Cardiovascular Institute, ${ }^{\mathrm{c}}$ Tokyo, Japan.

Disclosures: Authors have nothing to disclose with regard to commercial support.

Received for publication March 16, 2014; revisions received June 24, 2014; accepted

for publication July 8, 2014; available ahead of print Sept 22, 2014.

Address for reprints: Alexander Massmann, MD, Clinic of Diagnostic and

Interventional Radiology, Saarland University Medical Center, Kirrberger Straße,

66421 Homburg/Saar, Germany (E-mail: alexander.massmann@uks.eu).

$0022-5223 / \$ 36.00$

Copyright (c) 2014 by The American Association for Thoracic Surgery

http://dx.doi.org/10.1016/j.jtcvs.2014.07.053
}

has replaced surgery as the treatment of choice. This has been combined with insertion of uncovered stents into compromised aortic branches. ${ }^{1-5}$

In the past 10 years, the insertion of covered stents into the aortic true lumen (aTL) of the proximal descending aorta has become increasingly popular to seal the entry into the false lumen (FL). ${ }^{6-12}$ This concept has its limitations, for example, in the presence of several entries, which is not uncommon. In addition, it requires a constantly available, well-trained team and a stock of several aortic stent grafts to accommodate the variability of aortic sizes. Placement of aortic stent grafts requires large and stiff introducer systems. Given the texture of an acute dissected aortic wall and distal vessels, this may result in relevant vascular damage.

Malperfusion in CAADB is primarily caused by true lumen (TL) collapse. Reexpansion of the TL by uncovered stents should be effective in reestablishing visceral blood flow and has been successfully performed in the past. This approach requires only a $10 \mathrm{~F}$ introducer system and is therefore less traumatic than placement of stent grafts.

\section{OBJECTIVE}

The purpose of this study was to retrospectively evaluate the midterm clinical outcome after TL implantation of 


\section{Abbreviations and Acronyms \\ aTL = aortic true lumen \\ cAADB $=$ complicated acute aortic dissection Stanford type B \\ $\mathrm{CTA}=$ computed tomographic angiography \\ FL $\quad$ false lumen \\ SMA = superior mesenteric artery \\ TEVAR $=$ thoracic endovascular aortic/aneurysm repair \\ $\mathrm{TL}=$ true lumen}

uncovered bare-metal stents without proximal entry tear coverage for the treatment of visceral malperfusion and peripheral ischemia in cAADB.

\section{MATERIALS AND METHODS}

This was a consecutive series of 14 patients ( 3 female, 11 male; mean age, $58 \pm 11$ years [range, $43-72$ years]) with cAADB who were diagnosed clinically with possible visceral ischemia. After Institutional Review Board approval and patient's informed consent, all patients underwent primary interventional treatment for vital organ ischemia. The time interval between acute chest pain and onset of abdominal symptoms varied from 5 hours to 3 days. All patients had acute abdominal pain with gastrointestinal bleeding and/or diarrhea. In 6 patients, concomitant lower limb ischemia was present. Renal ischemia was suspected in 6 patients because of anuria and increased creatinine levels. Abnormal laboratory test results (increased lactate, creatine kinase, lipase, amylase, and transaminase levels) indicating ischemia were present in all patients (Table 1). cAADB was confirmed by initial contrast-enhanced electrocardiography-gated computed tomography (computed tomographic angiography [CTA]) of the aorta including the supraaortic, iliac, and femoral arteries. In all cases, CTA showed marked aTL compression (Figure 1). Relevant narrowing of the visceral and/or iliac TL was observed in 9 patients.

\section{Endovascular Treatment}

Endovascular treatment was performed within 2 to 6 hours after the onset of abdominal symptoms in all patients. No patient underwent treatment later than this time interval. All patients underwent catheter angiography via transfemoral access. The puncture site was chosen according to the extent and course of dissection in the CTA to ensure TL access. A pigtail catheter was placed into the abdominal aorta. The position inside the TL was verified angiographically. In 1 case, puncture of the FL occurred initially; subsequent TL access was established via the contralateral femoral artery. Transbrachial access was not necessary in any case.

The catheter was then advanced into the aortic arch for aortography. In all 10 patients with relevant aTL compression as documented by CTA, reduced flow to the TL was verified angiographically. The celiac axis and superior mesenteric artery (SMA) arose from the collapsed TL resulting in the typical angiographic sign of floating visceral arteries (Figure 2).

In a further 4 cases, visceral malperfusion was caused by extension of the dissection into the SMA or celiac trunk. The TL of the visceral arteries was considerably stenosed by an enlarged FL.

In 5 patients, delayed or absent blood flow was observed in the common iliac arteries.

Stent implantation into the aorta was performed in all 10 patients with aTL compression to establish sufficient flow to visceral and iliac vessels (Table 1).
Localization for stent placement and sizing was based on CTA and angiography. CTA was analyzed for morphology and to establish the diameter of the aTL and aortic FL. Angiography was used for hemodynamic assessment of functional compromise.

For aTL stenting, self-expanding bare-metal nitinol stents were used (sinus-XL 10F, OptiMed Medizinische Instrumente GmbH, Ettlingen, Germany), which are characterized by a closed-cell design with large mesh size and high radial force. The dimension of 1 single cell is about 3 to $6 \mathrm{~mm}$ by $9 \mathrm{~mm}$ (width $\times$ length) depending on the stent diameter $(18-36 \mathrm{~mm})$.

The stent size for aTL was chosen according to one quarter to one third of the original cross-sectional aortic diameter including the TL and FL. This intentional undersizing in relation to the original aortic diameter was used to guarantee complete stent expansion. Only full stent expansion in the optimal configuration assures the highest radial force.

Depending on the cranial extent of aTL compression, the first stent was placed in the thoracoabdominal position with the distal end just above the origin of the visceral arteries $(n=6)$. One patient underwent stent implantation into the middle descending thoracic aorta because of severe aTL compression at that level. Two patients underwent stent implantation just at the level of the visceral artery ostia. No compromise of visceral artery inflow was observed by this overstenting of the visceral artery lumina. This was true for the acute setting as well as for the follow-up at 2 years.

Inadequate blood flow to the inferior mesenteric and iliac arteries was observed in 4 patients after insertion of a thoracoabdominal stent. This was caused by aTL compression of the infrarenal aorta. These 4 patients underwent additional infrarenal aortic stent implantation.

In order to ascertain complete expansion, all stents were dilated up to the nominal stent diameter. Complete stent expansion was verified by fluoroscopy in orthogonal planes. Angiography was used to evaluate increased blood flow.

Stenting of compromised visceral arteries was performed with balloon-expandable cobalt-chromium stents $(\mathrm{n}=5)$ (Palmaz-Blue; Cordis, Diegem, Belgium) in 4 patients. Stent oversizing was typically $10 \%$. Additional stents were placed distal to the renal arteries or aorto-iliacal in the case of hemodynamically relevant stenosis as judged by clinically apparent lower limb ischemia.

Early in the series, additional infrarenal fenestration of the dissection was performed $(\mathrm{n}=4)$ for decompression of severe compromised aTL.

Access to the FL was obtained using a right-angled $5 \mathrm{~F}$ catheter with a 0.89 -mm $\left(0.035\right.$-inch) stiff guidewire. ${ }^{13}$ The hard end of the guidewire was used to perforate the dissection membrane under fluoroscopic guidance. After verification of access to the FL by angiography, balloon dilation (diameter $10 \mathrm{~mm}$ ) was performed in order to expand the perforation.

After intervention, all patients were transferred to an intensive care unit. Close clinical monitoring focused on persistent or recurrent abdominal pain or limb ischemia.

Postoperative imaging included ultrasonography of the visceral and iliac arteries, and CTA after 1 week, 1, 3, and 6 months, and then every 12 months.

Data were analyzed using GraphPad Prism 6.0d for Mac (GraphPad Software, Inc, La Jolla, Calif). Continuous variables were evaluated using the Student $t$ test.

\section{RESULTS}

Ten patients underwent implantation of 14 selfexpandable bare-metal stents (sinus-XL 10F and sinus-Repo-Visual 6F, OptiMed; Bard LUMINEXX 6F, C.R. Bard, Inc, Karlsruhe, Germany; WALLSTENT, Boston Scientific, Marlborough, Mass) into the thoracoabdominal aorta (Table 2, Figures 3 and 4). In addition, 
TABLE 1. Laboratory results for the patients with complicated type B dissection

\begin{tabular}{lccrcc}
\hline & \multicolumn{2}{c}{ Preintervention } & & \multicolumn{2}{c}{ Maximum } \\
\cline { 2 - 3 } \multicolumn{1}{c}{ Laboratory values [normal limits] } & Range & Mean \pm SD & & Range & Mean \pm SD \\
\hline Creatine kinase $[<167 \mathrm{U} / \mathrm{L}]$ & $30-9853$ & $1212 \pm 3066$ & & $110-126493$ & $29,034 \pm 40,380$ \\
Lactic dehydrogenase $[<289 \mathrm{U} / \mathrm{L}]$ & $193-1300$ & $372 \pm 354$ & & $239-10,494$ & $2637 \pm 3506$ \\
Lactate $[<2.2 \mathrm{mmol} / \mathrm{L}]$ & $0.3-2.0$ & $1.6 \pm 0.8$ & & $1.7-15$ & $6.6 \pm 5.1$ \\
Creatinine $[<1.2 \mathrm{mg} / \mathrm{dL}]^{*}$ & $0.49-1.9$ & $1.3 \pm 0.5$ & & $0.9-15.5$ & $4.6 \pm 4.7$ \\
Estimated glomerular filtration rate $\left[\mathrm{mL} / \mathrm{min} / 1.73 \mathrm{~m}^{2}\right]^{*}$ & $35.8-110.3$ & $58.6 \pm 24.8$ & & $3.4-60.9$ & $24.5 \pm 18.6$ \\
C-reactive protein $[<3 \mathrm{mg} / \mathrm{L}]$ & $0.8-42.5$ & $10.0 \pm 13.2$ & & $18.2-405.2$ & $197.6 \pm 118.6$ \\
\hline
\end{tabular}

Because of different kinetics for the different parameters, baseline and maximum data are shown. $S D$, standard deviation. *Patients with dialysis-dependent chronic renal failure are excluded.

9 self-expandable bare-metal stents (Luminexx-6F, Bard; SinusVisualRepo-6F, Optimed) were placed into the aortoiliac arteries (Table 2).

Fenestration of the dissection membrane was performed in 4 patients. Satisfactory relief of visceral ischemia was not obtained by fenestration alone in any patient. In these cases, and in 1 patient with extreme intramural hematoma of the thoracoabdominal aorta, additional visceral artery stenting sufficiently improved visceral perfusion; 4 patients underwent implantation of 5 balloonexpandable cobalt-chromium stents (Palmaz-Blue) into the mesenteric $(n=3)$ or renal $(n=2)$ arteries after aortic fenestration. Details of the number, location, and stent type for each patient are given in Table 2.

The primary technical success rate for stent implantation was $100 \%$. No peri-interventional complications occurred. Follow-up CTA after 1 week revealed collapse of 4 aortic stents (sinus-XL 10F; diameter, $9 \mathrm{~mm}, 20 \mathrm{~mm}$, and 2 of $25 \mathrm{~mm}$; length, $100 \mathrm{~mm}$ ) in 4 patients (Figure 5). Two of these patients received aortic fenestration for normalization of the pressure gradient between aTL and FL before stent implantation.

Successful catheter angiography with balloon dilation was performed on all 4 stents. Nevertheless, all patients showed recurrent collapse on follow-up CTA 1 month later. In 1 of these stents, a fracture with complete disruption of the stent struts was documented 3 months later (Figure 6).

All patients stabilized and remained asymptomatic without the need for reintervention during the initial hospital stay. Stenting resulted in marked clinical improvement in all patients in the following 24 hours. There was complete absence of abdominal pain and cessation of claudication. No patients required additional interventional or surgical therapy related to aortic dissection and its sequelae during follow-up, which ranged from 6 to 124 months (mean \pm standard deviation, $45 \pm 47$ months).

All patients with anuria caused by acute renal failure $(\mathrm{n}=4)$ required dialysis; in 2 patients renal function improved within a week. Two additional patients were already in dialysis-dependent chronic renal failure before the onset of aortic dissection. There was no patient with new onset of chronic renal failure after interventional treatment. Objective relief of visceral ischemia, for example, normalization of lactic acidosis, was ascertained by laboratory tests in all patients, including these with previous chronic kidney disease.

There was no significant growth of the descending thoracic aorta during follow-up. One patient showed an

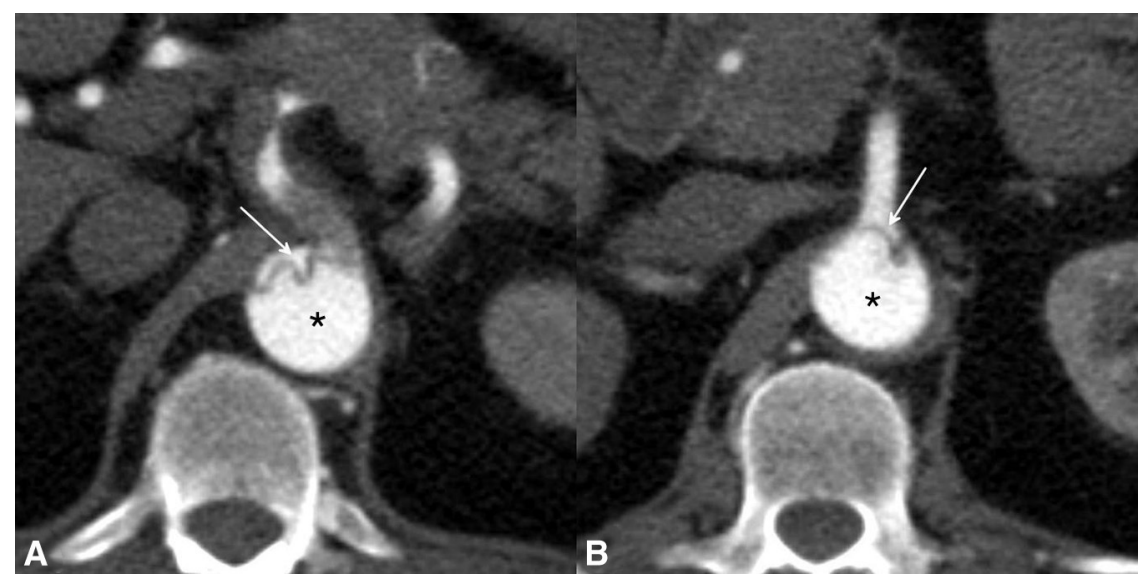

FIGURE 1. A, Computed tomographic angiography shows a very small true lumen (arrow) of the abdominal aorta, which is nearly completely compressed by the false lumen (asterisk). The dissection extends into the celiac trunk. B, The superior mesenteric artery ostium is covered by the dissection membrane. The aortic true lumen is almost completely collapsed (arrow). 


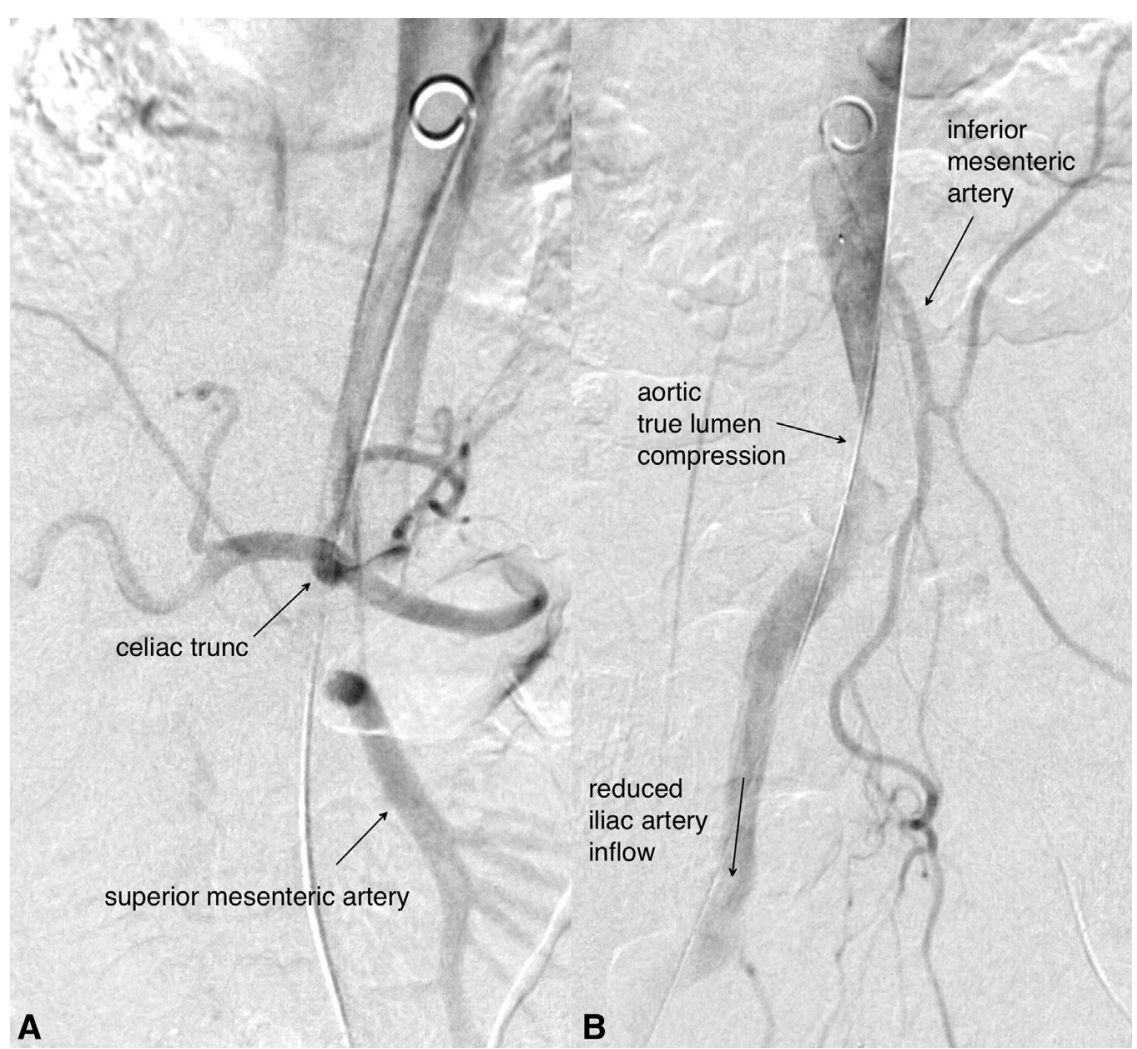

FIGURE 2. A, Catheter angiography after contrast medium injection into the aortic true lumen results in the typical appearance of floating visceral arteries. $\mathrm{B}$, Contrast injection into the aortic true lumen of the infrarenal aorta shows a high-grade stenosis due to almost complete compression of the true lumen. Inflow into the iliac arteries is markedly reduced.

increase of $9 \mathrm{~mm}$ in the diameter of the abdominal aorta at the level of the celiac trunk ( $27 \mathrm{~mm}$ vs $36 \mathrm{~mm}$ ). In all other cases the diameter of the abdominal aorta remained stable (Figure 7). All FLs were patent without any relevant thrombosis. Ultrasonography and CTA showed patent perfusion of the SMA in all patients and at every time point.

Two patients with enlargement of the ascending thoracic aorta underwent replacement of the aortic valve and ascending aorta for an ascending aortic aneurysm.

Freedom from aortic rupture or replacement of the descending aorta was $100 \%$. There were no neurologic complications (eg, paraplegia, transient ischemic attack, or stroke). All patients are alive and well at the time of this report.

\section{DISCUSSION}

cAADB is characterized by rupture or impending rupture, malperfusion, refractory pain, or hypertension. Organ ischemia is caused by obstruction of visceral or peripheral arteries by the pressurized TL and dissection membrane. Organ ischemia occurs in $30 \%$ to $50 \%$ of all aortic dissections, and ischemia of the abdominal viscera is one of the main causes of death in cAADB. The primary objective is to reestablish sufficient aortic, visceral, and

TABLE 2. Number, type, and location of stent implantations in patients with complicated type B dissection

\begin{tabular}{|c|c|c|c|c|c|}
\hline Region & Patients & Stents/patient & Stent type & Stents & Stent dimension \\
\hline \multirow[t]{3}{*}{ Thoracoabdominal aorta } & 9 & Up to 4 & sinus-XL 10F (OptiMed) & 12 & Diameter 7-28 mm \\
\hline & & & sinus-Repo-Visual 6F (OptiMed) & 1 & Length $40-100 \mathrm{~mm}$ \\
\hline & & & WALLSTENT (Boston Scientific) & 1 & \\
\hline \multirow[t]{2}{*}{ Aorto-iliacal arteries } & 5 & Up to 4 & LUMINEXX 6F (Bard) & 8 & Diameter 8-10 mm \\
\hline & & & sinus-Repo-Visual 6F (OptiMed) & 1 & Length $40-80 \mathrm{~mm}$ \\
\hline \multirow[t]{4}{*}{ Visceral arteries } & 4 & Up to 2 & Palmaz-Blue 4F (Cordis) & 5 & Diameter 5-7 mm \\
\hline & & & Celiac trunk $(\mathrm{n}=2)$ & & Length $18-24 \mathrm{~mm}$ \\
\hline & & & Superior mesenteric artery $(\mathrm{n}=1)$ & & \\
\hline & & & Renal artery $(n=2)$ & & \\
\hline
\end{tabular}




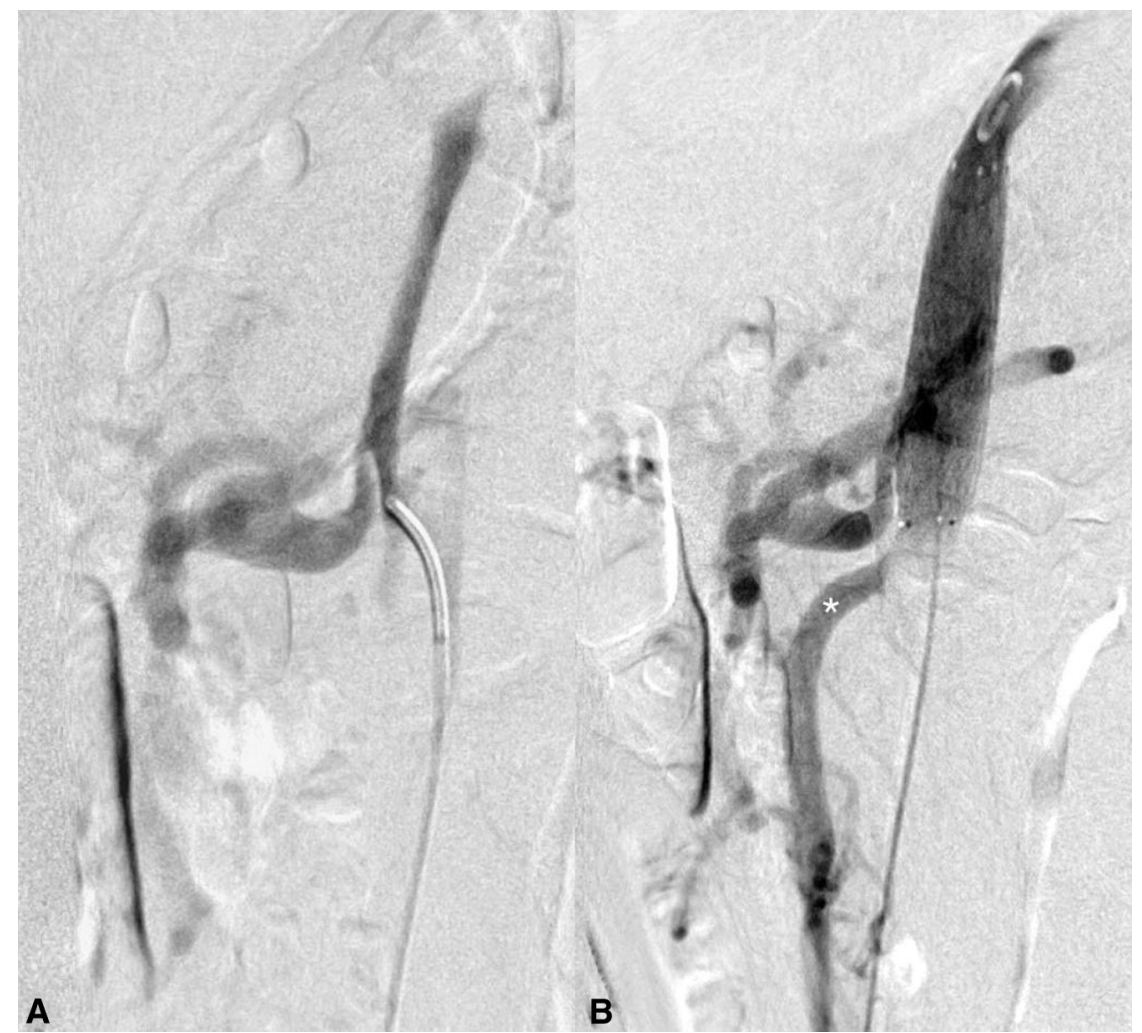

FIGURE 3. A, Angiography reveals a complete occlusion of the superior mesenteric artery originating from the complete compression of the true aortic lumen. B, Stent implantation into the true aortic lumen results in a markedly improved inflow into the superior mesenteric artery (asterisk) despite stent placement over the superior mesenteric artery ostium. Because of impaired infrarenal blood flow, additional infrarenal stenting was performed.

peripheral artery blood flow by reexpansion of the compressed TL. Optimal management of cAADB is still controversial. Traditional treatment has been surgery with either replacement of the dissected proximal thoracic descending aorta or fenestration. ${ }^{14}$ Both approaches have been associated with considerable morbidity and mortality. ${ }^{8,9,14,15}$ Descending aortic replacement has resulted in greater than $30 \%$ mortality. Surgical fenestration has not universally resulted in relief of end-organ ischemia ${ }^{16,17}$ and has also been associated with high mortality. ${ }^{9}$ Operative mortality in the presence of visceral ischemia remains as high as $70 \%$ to $80 \%$ either due to rupture or visceral ischemia. $^{18}$

Endovascular treatment has been introduced in the last 2 decades $^{6}$ including implantation of stent grafts, $, 810,19$ catheter-based fenestration, ${ }^{13,17,20}$ or stenting of aortic

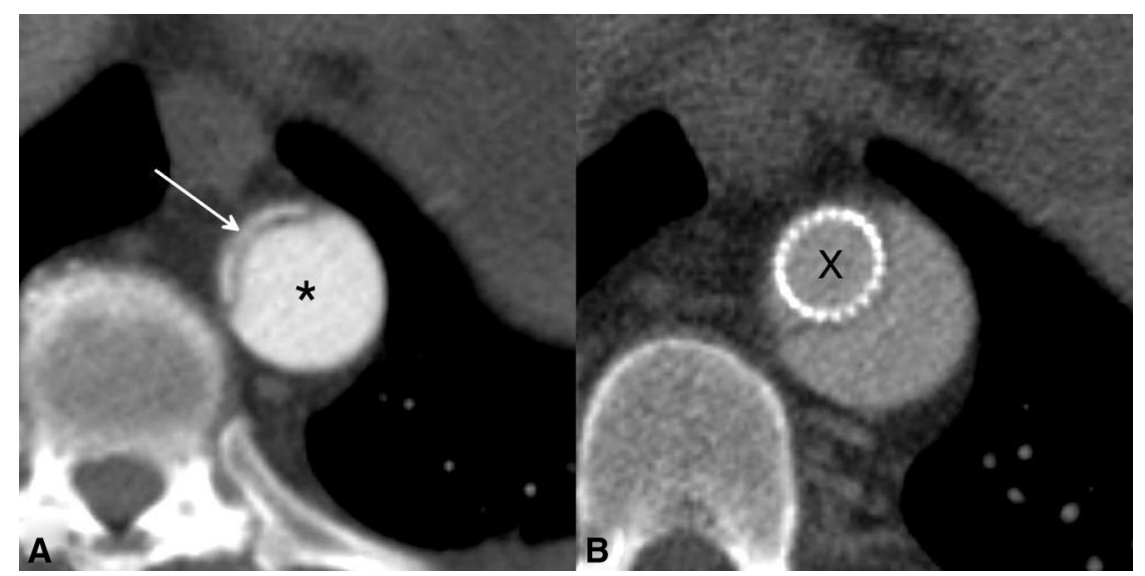

FIGURE 4. A, Computed tomographic angiography depicts a slitlike configuration of the true aortic lumen, which is compressed by a large false lumen (asterisk). B, Adapted stent implantation achieved a perfused true lumen (X) of about one quarter to one third of the original aortic diameter. 


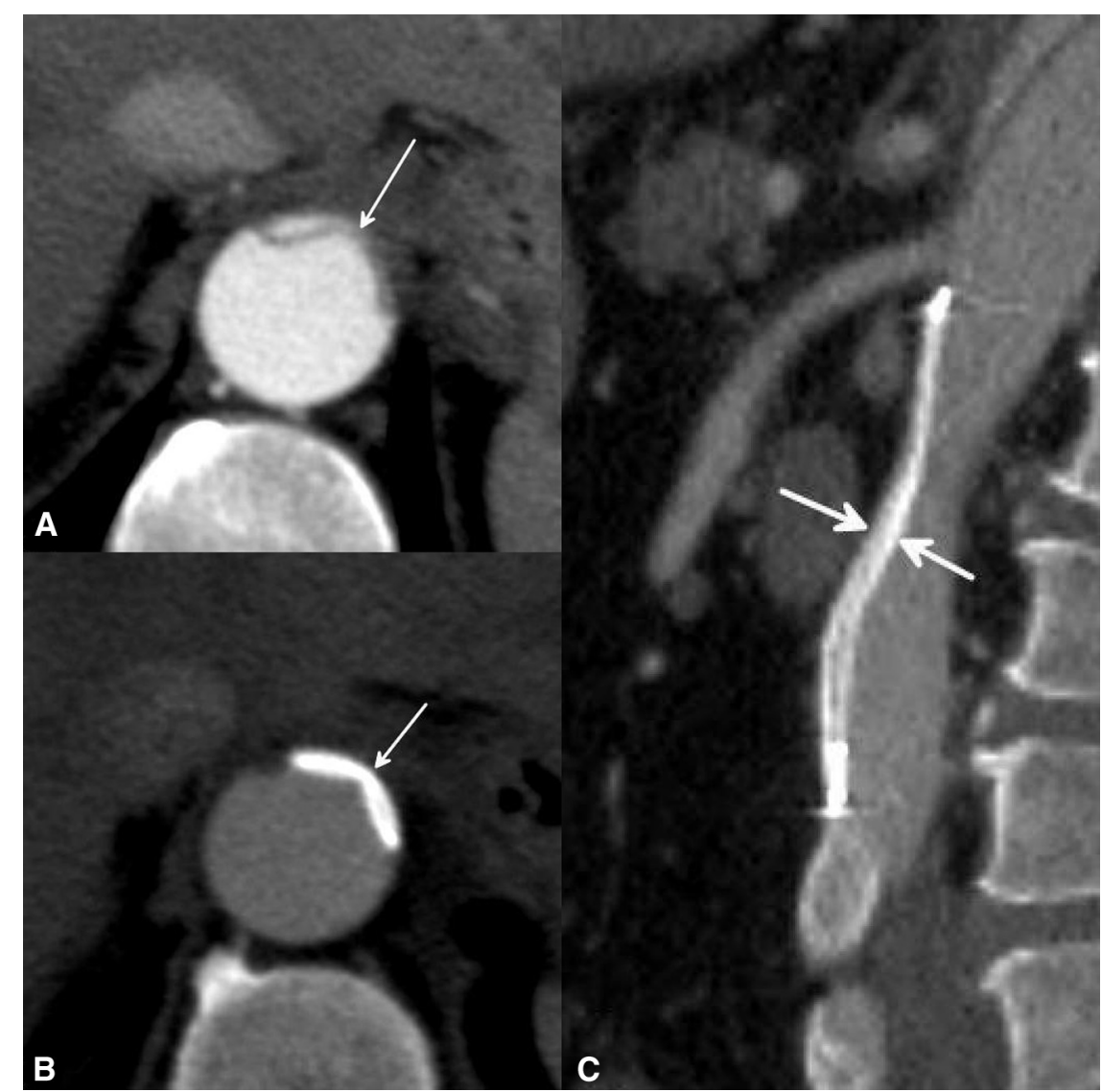

FIGURE 5. A, Computed tomographic angiography initially reveals a compressed aortic true lumen. After stent implantation into the aortic true lumen, computed tomographic angiography 2 weeks later shows stent collapse (arrows) in transverse (B) and sagittal (C) views.

branches. $^{7}$ The objective is restoration and stabilization of blood flow and dissection. Intimal flap mobility, aortic or branch vessel obstruction, and perfusion of visceral organs must be reproducibly improved without increasing the risk of aortic rupture. ${ }^{7,10,17,20-22}$ In this context, the use of thoracic endovascular aortic/aneurysm repair (TEVAR) has been proposed to be beneficial for aortic remodeling; but TEVAR had no positive effect on 2-year survival. ${ }^{19}$ Nevertheless, the main short-term goal is to establish and ensure a patent TL for adequate blood supply to the visceral arteries and lower extremities as fast as possible. TEVAR attempts to achieve this goal by closing the proximal entry; fenestration of the dissection membrane creates distal reentries allowing the pressure gradient between the TL and FL to be minimized, and stenting is meant to eliminate the luminal occlusion of compressed aortic branches.

Using such endovascular approaches in an often individualized fashion seems to have a beneficial effect compared with open surgery. ${ }^{8,12,15,23}$ Aortic stent graft implantation, fenestration, and stenting of the affected aortic branches have thus become generally preferred in cAADB. ${ }^{8,23-25}$ However, they still have drawbacks. The use of interventional fenestration for the treatment of malperfusion has had only variable success in
cAADB. ${ }^{13,16,17}$ Procedure-associated severe complications include aortic perforation and rupture. ${ }^{26,27}$ In the past, we have not been impressed with the clinical results. In our current series, we used it in selected early cases, and again its effect was not reproducible. With the positive results of uncovered stenting, we have largely aborted interventional/surgical fenestration. Stenting of aortic branches has been primarily successful in limited occlusion of these vessels. It does not, however, treat a more general aTL collapse, which is frequently seen in cAADB.

After the advent of TEVAR, simple stenting of the aorta and visceral arteries was abandoned in many centers. TEVAR has often been successful by closing the entry site into the FL. Thereby, the pressure of the FL and TL compression were minimized improving blood flow through the aTL and into the visceral arteries. Most likely due to the presence of reentries, its effect on aTL collapse has not always been predictable. TEVAR may be difficult to position in the presence of preexisting dilatation of the descending aorta, which is not infrequent in cAADB. Patency of the supraaortic vessels has to be maintained and sometimes requires a hybrid procedure to ensure perfusion of all supraaortic vessels, especially if dissection 


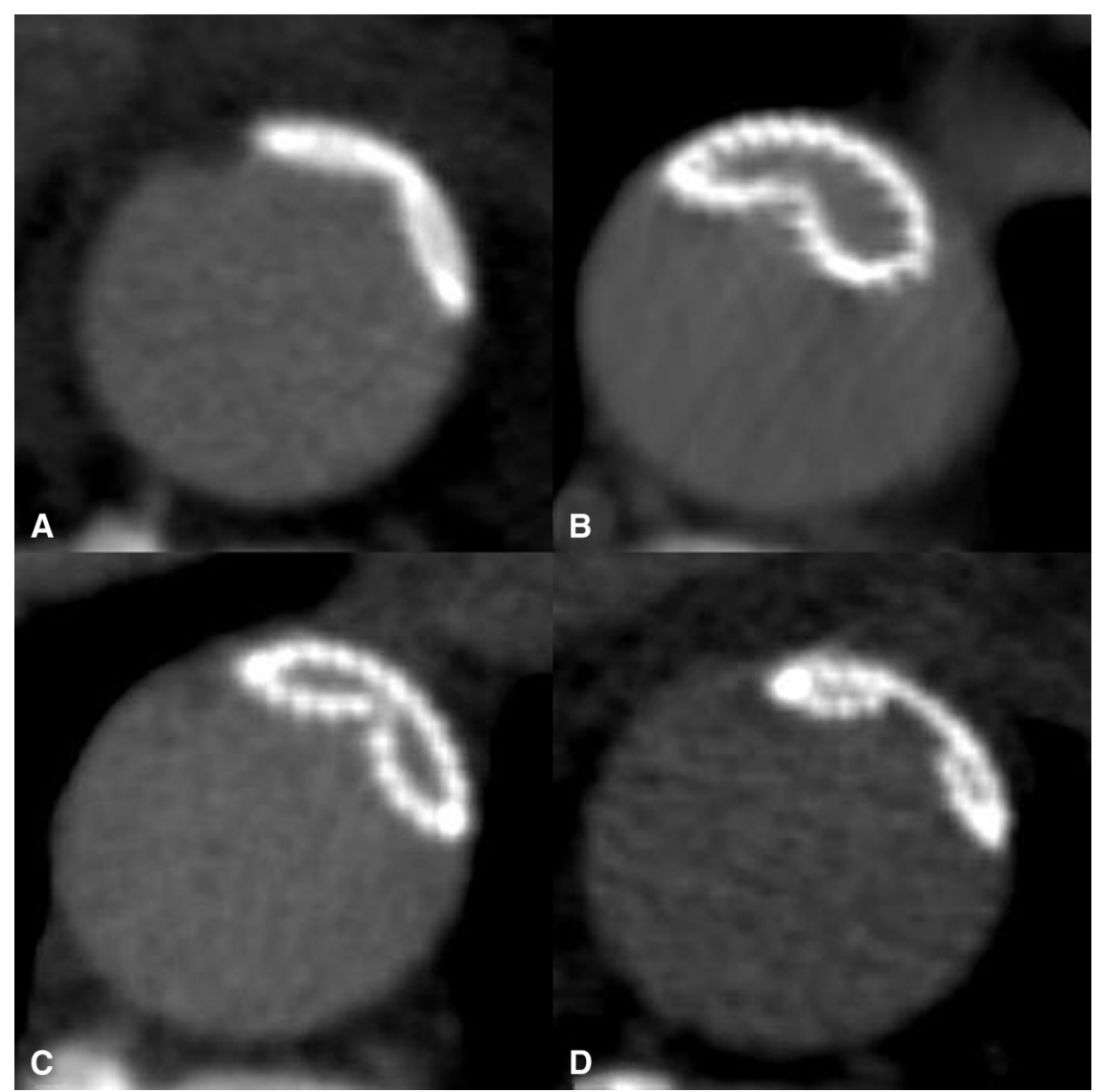

FIGURE 6. A, Follow-up computed tomographic angiography of the patient in Figure 5, 2 weeks after stent implantation revealed stent collapse. B, Balloon angioplasty resulted in a partial reexpansion of the stent. C, Follow-up computed tomographic angiography 4 weeks after reexpansion documents recollapse. D, Follow-up computed tomographic angiography another 4 weeks after recollapse depicts complete disruption of the stent struts. Despite this unsatisfactory morphologic result, the patient did not complain of clinical symptoms.

involves the aortic arch. In addition, spinal ischemia is a known risk factor with TEVAR. Stent grafts have the disadvantage of requiring relatively stiff and large introducer systems. Retrograde aortic dissection involving the ascending aorta and perforations have been reported, which were most likely related to the fragile texture of the sub-/acutely dissected aorta. ${ }^{28}$

Even after correct stent graft implantation, aTL collapse may persist despite obliteration of the proximal thoracic aortic entry tear. ${ }^{20}$ Although current interventional approaches have been successful, current techniques still seem suboptimal. An optimal approach should involve less invasive introducer systems of smaller diameter. Inadequate iliac access vessels with atherosclerotic disease are a technical contraindication for TEVAR.

If there is a need for additional aortic and visceral artery stenting after TEVAR, the question has to be asked whether TEVAR offers any benefits over the initial and sole treatment of distal aortic and visceral artery stenting, especially when the additional potential complications of TEVAR compared with stenting alone are taken into consideration.
Because TL compression seems to be of central importance in cAADB with visceral malperfusion, we hypothesized that TL stenting should be effective in alleviating visceral ischemia. The above-mentioned disadvantages of stent grafts prompted us to evaluate the application of uncovered stents for the treatment of visceral malperfusion. Uncovered stents have more flexible delivery systems with small low-profile $10 \mathrm{~F}(3 \mathrm{~mm})$ delivery systems. Therefore, insertion and positioning is atraumatic and technically relatively easy. Covered stent grafts require a diameter of 20 to $24 \mathrm{~F}$ (7-8 $\mathrm{mm})$, which results in a much higher shear stress to iliac access vessels, especially in unfavorable tortuous anatomy.

Covering of abdominal branch vessels is tolerable, if needed to alleviate malperfusion. No reduced inflow to visceral arteries was observed in any of our cases, due to the relatively wide mesh structure of the stent struts. Nonetheless, we usually try to avoid aortic stent placement over visceral artery ostia.

We observed 4 cases of stent collapse after decompressing a slitlike compressed aTL at the thoracoabdominal 


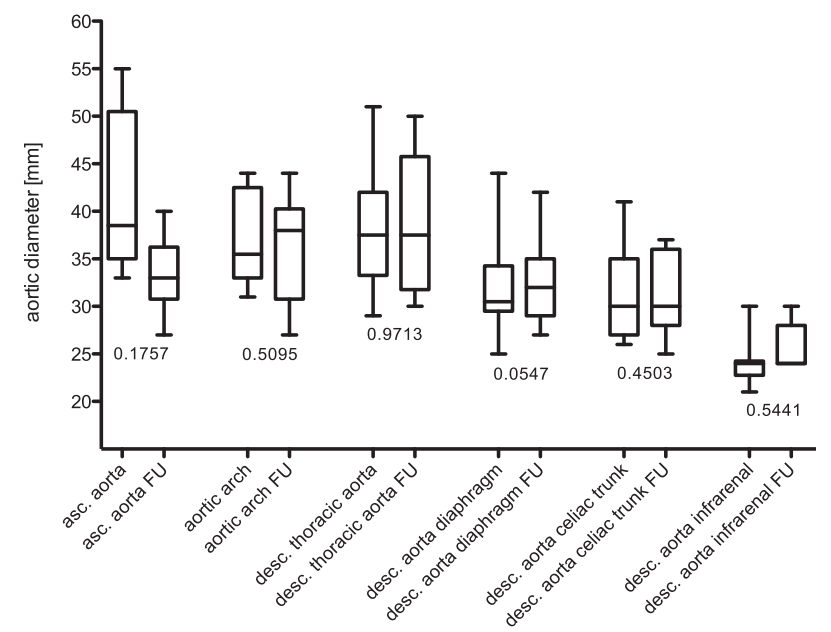

FIGURE 7. Aortic diameter on initial and follow-up computed tomographic angiography: box and Whiskers plot ( $\mathrm{min} / \mathrm{max} / 25$ th percentiles) with $P$ values. asc, Ascending; $F U$, follow-up; desc, descending.

level. One stent reexpanded spontaneously. Reexpansion by balloon angioplasty was temporarily successful in 3 , but recollapse was seen a few weeks later on follow-up computed tomography. The underlying cause for this stent collapse is most likely related to inadequate radial forces of the stent. One the one hand, there is a perpetual pulsatile pressure in the FL because the primary entry tear is not treated. Despite additional fenestration for normalization of the pressure gradient, stent collapse occurred in 2 of these 4 patients. Even though the exact mechanism could not be clarified, its occurrence did not lead to any negative clinical results, possibly because of preservation of sufficient blood flow. The reason for the positive clinical results may be related to preservation of sufficient flow in the rounded corners of the collapsed stent (Figure 5, A). Alternatively, stent collapse may have developed gradually, allowing for collateral blood flow to develop.

In order to minimize the occurrence of stent collapse, nowadays we choose aortic stents with a smaller diameter. This intentional undersizing in relation to the original aortic diameter allows complete stent expansion resulting in a rounded shape and optimal radial force. This configuration should allow the stents to bear the highest radial pressure. The radial force of an incompletely inflated stent will always be much lower than a perfectly expanded stent.

Determination of appropriate stent size is difficult. According to our experience, a stent diameter of about one quarter to one third of the original aortic diameter is favorable. Oversizing should be avoided; the stent should not be chosen to achieve the original diameter of the aorta. Instead, it should only open the TL for a break through of the initial ischemic phase.

Usually, stent undersizing can lead to stent displacement. In aortic dissection, however, the forces of the dissection membrane are sufficient to secure an undersized stent in place.

In addition, interventional treatment leads to stabilization of the descending aortic diameter. The diameter of the abdominal aorta showed no statistically significant increase after a mean follow-up of 3 years. Only replacement of the ascending aorta and aortic valve was necessary in 2 patients for concomitant ascending aortic aneurysm. No thrombosis of the aortic FL occurred. Although this, in conjunction with remodeling, is one of the arguments for a TEVAR, we could not see any disadvantage of a still-perfused FL. Neither increasing aortic aneurysms nor ulcers were seen during follow-up. The results are at least as effective as covered stent placement into the aorta or aortic branches alone or as an adjunct to percutaneous fenestration. ${ }^{5,25,29,30}$

The limitations of this study are mainly its small sample size and retrospective nature. It is impossible to say which endovascular treatment is best for cAADB from our results. The aggressive approach to treating potentially ischemic regions is consistent with our intention to avoid any delay in treatment, which may lead to irreversible damage. All patients were treated before development of acidosis and shock, and we contribute our favorable results to the fact that very early intervention avoided the fatal scenario of intestinal gangrene.

The positive results stress the point that even an older technique like uncovered stent placement may be a viable alternative to stent grafts. This is especially important for those hospitals where logistic requirements cannot be fulfilled and sufficient knowledge of aortic stent graft placement is not available.

In conclusion, thoracoabdominal uncovered stent implantation is a technically feasible and effective therapy in cAADB. The study provides a strong case for very early intervention in patients with acute aortic dissection if there is a suspicion of visceral ischemia. Uncovered stent implantation provided successful relief of acute visceral ischemia or claudication and yielded a similar if not better clinical outcome during midterm follow-up compared with the literature data on TEVAR. Stent size should be adapted to about one third of the original aortic diameter. Stents with high radial force should be used to avoid stent collapse. Uncovered stent implantation did not lead to progressive aortic expansion at midterm follow-up.

\section{References}

1. Chavan A, Rosenthal H, Luthe L, Pfingsten S, Kutschka I, Easo J, et al. Percutaneous interventions for treating ischemic complications of aortic dissection. Eur Radiol. 2009;19:488-94.

2. Lauterbach SR, Cambria RP, Brewster DC, Gertler JP, Lamuraglia GM, Isselbacher EM, et al. Contemporary management of aortic branch compromise resulting from acute aortic dissection. J Vasc Surg. 2001;33:1185-92.

3. Beregi J-P, Haulon S, Otal P, Thony F, Bartoli J-M, Crochet D, et al. Endovascular treatment of acute complications associated with aortic dissection: midterm results from a multicenter study. J Endovasc Ther. 2003; 10:486-93. 
4. Slonim SM, Nyman UR, Semba CP, Miller DC, Mitchell RS, Dake MD. True lumen obliteration in complicated aortic dissection: endovascular treatment. Radiology. 1996;201:161-6.

5. Williams DM, Lee DY, Hamilton BH, Marx MV, Narasimham DL, Kazanjian SN, et al. The dissected aorta: percutaneous treatment of ischemic complications-principles and results. J Vasc Interv Radiol. 1997;8:605-25.

6. Fann JI, Sarris GE, Mitchell RS, Shumway NE, Stinson EB, Oyer PE, et al. Treatment of patients with aortic dissection presenting with peripheral vascular complications. Ann Surg. 1990;212:705-13.

7. Sfyroeras GS, Rubio V, Pagan P, Diethrich EB, Rodriguez JA. Endovascular management of malperfusion in acute type B aortic dissections. J Endovasc Ther. 2011;18:78-86.

8. Fattori R, Tsai TT, Myrmel T, Evangelista A, Cooper JV, Trimarchi S, et al. Complicated acute type B dissection: is surgery still the best option? A report from the International Registry of Acute Aortic Dissection. JACC Cardiovasc Interv. 2008;1:395-402.

9. Laas J, Heinemann M, Schaefers HJ, Daniel W, Borst HG. Management of thoracoabdominal malperfusion in aortic dissection. Circulation. 1991; 84(5 Suppl):III20-4.

10. Svensson LG, Kouchoukos NT, Miller DC, Bavaria JE, Coselli JS, Curi MA, et al. Expert consensus document on the treatment of descending thoracic aortic disease using endovascular stent-grafts. Ann Thorac Surg. 2008;85(1 Suppl):S1-41.

11. Swee W, Dake MD. Endovascular management of thoracic dissections. Circulation. 2008;117:1460-73.

12. Zeeshan A, Woo EY, Bavaria JE, Fairman RM, Desai ND, Pochettino A, et al. Thoracic endovascular aortic repair for acute complicated type B aortic dissection: superiority relative to conventional open surgical and medical therapy. J Thorac Cardiovasc Surg. 2010;140(6 Suppl):S109-15; discussion S142-6.

13. Hartnell GG, Gates J. Aortic fenestration: a why, when, and how-to guide. Radiographics. 2005;25:175-89.

14. Heinemann MK, Buehner B, Schaefers HJ, Jurmann MJ, Laas J, Borst HG. Malperfusion of the thoracoabdominal vasculature in aortic dissection. $J$ Card Surg. 1994;9:748-55; discussion 755-7.

15. Wilkinson DA, Patel HJ, Williams DM, Dasika NL, Deeb GM. Early open and endovascular thoracic aortic repair for complicated type B aortic dissection. Ann Thorac Surg. 2013;96:23-30; discussion 230.

16. Pradhan S, Elefteriades JA, Sumpio BE. Utility of the aortic fenestration technique in the management of acute aortic dissections. Ann Thorac Cardiovasc Surg. 2007;13:296-300.

17. Midulla M, Renaud A, Martinelli T, Koussa M, Mounier-Vehier C, Prat A, et al. Endovascular fenestration in aortic dissection with acute malperfusion syndrome: immediate and late follow-up. J Thorac Cardiovasc Surg. 2011; 142:66-72.
18. Von Kodolitsch Y, Schwartz AG, Nienaber CA. Clinical prediction of acute aortic dissection. Arch Intern Med. 2000;160:2977-82.

19. Nienaber CA, Rousseau H, Eggebrecht H, Kische S, Fattori R, Rehders TC, et al Randomized comparison of strategies for type B aortic dissection: the INvestigation of STEnt Grafts in Aortic Dissection (INSTEAD) trial Circulation. 2009;120:2519-28.

20. Slonim SM, Miller DC, Mitchell RS, Semba CP, Razavi MK, Dake MD. Percutaneous balloon fenestration and stenting for life-threatening ischemic complications in patients with acute aortic dissection. J Thorac Cardiovasc Surg. 1999;117:1118-26.

21. Nienaber CA, Kische S, Zeller T, Rehders TC, Schneider H, Lorenzen B, et al Provisional extension to induce complete attachment after stent-graft placemen in type B aortic dissection: the PETTICOAT concept. J Endovasc Ther. 2006;13: 738-46.

22. Fattori R, Botta L, Lovato L, Biagini E, Russo V, Casadei A, et al. Malperfusion syndrome in type B aortic dissection: role of the endovascular procedures. Acta Chir Belg. 2008;108:192-7.

23. Szeto WY, McGarvey M, Pochettino A, Moser GW, Hoboken A, Cornelius K, et al. Results of a new surgical paradigm: endovascular repair for acute complicated type B aortic dissection. Ann Thorac Surg. 2008;86:87-93; discussion 93-4.

24. Eggebrecht H, Lönn L, Herold U, Breuckmann F, Leyh R, Jakob HG, et al Endovascular stent-graft placement for complications of acute type B aortic dissection. Curr Opin Cardiol. 2005;20:477-83.

25. Fattori R, Nienaber CA, Rousseau H, Beregi J-P, Heijmen R, Grabenwöger M, et al. Results of endovascular repair of the thoracic aorta with the Talent Thoracic stent graft: the Talent Thoracic Retrospective Registry. J Thorac Cardiovasc Surg. 2006;132:332-9.

26. Lookstein RA, Mitty H, Falk A, Guller J, Nowakowski FS. Aortic intima dehiscence: a complication of percutaneous balloon fenestration for aortic dissection. J Vasc Interv Radiol. 2001;12:1347-50.

27. Maynar M, Rostagno R, Zander T, Qian Z, Llorens R, Zerolo I, et al. Intima dehiscence in the abdominal aorta following balloon fenestration for type B dissection. J Endovasc Ther. 2005;12:103-9.

28. Kolluri R, Fowler B, Nandish S. Vascular access complications: diagnosis and management. Curr Treat Options Cardiovasc Med. 2013; 15:173-87.

29. Dake MD, Kato N, Mitchell RS, Semba CP, Razavi MK, Shimono T, et al. Endovascular stent-graft placement for the treatment of acute aortic dissection. N Engl J Med. 1999;340:1546-52.

30. Nienaber CA, Fattori R, Lund G, Dieckmann C, Wolf W, von Kodolitsch Y, et al Nonsurgical reconstruction of thoracic aortic dissection by stent-graft placement N Engl J Med. 1999;340:1539-45. 